\title{
Microsimulation Study of the Release of Pneumonic Plague and Smallpox on a Synthetic Civilian Population
}

\author{
$\underline{\text { A Green }}^{\mathrm{a}}$, I Piper ${ }^{\mathrm{a}}$, D Keep ${ }^{\mathrm{a}}$ \\ ${ }^{a}$ School of Computer Science and Software Engineering, University of Wollongong, Northfields Ave, \\ Wollongong, NSW 2500 \\ Email:tgreen@uow.edu.au
}

\begin{abstract}
:
The confrontation in Syria during 2013 is an ongoing cause for concern regarding the potential use of chemical and biological weapons. There have been reports of the use of chemical weapons including Sarin (BBC, 2013a) which UN chemical weapons inspectors are investigating (BBC, 2013b). If chemical weapons have been used by either side, then the potential use of biological weapons cannot be disregarded. In addition to stockpiles of chemical weapon (BBC 2013c), Syria is thought to have stockpiles of a number of biological agents including anthrax, plague, tularaemia, botulinium, smallpox and cholera (Gordon, 2007). Some groups sympathetic to $\mathrm{Al}$ Qaeda might also have access to some of these through their terrorist networks. Because these weapons can have a substantial impact beyond the immediate conflict zone, there are serious questions about how best to respond efficiently to their use and manage their impacts.
\end{abstract}

One such concern is whether the response to an attack involving a single agent would be the same as when more than one agent is used. There are indications that infectious diseases which promote cytokine response can have a protecting effect on infection with a second disease (Graham et al., 2007, Barton et al, 2007). Plague affects the innate immune system by suppressing cytokine responses (Li et al., 2008) while smallpox activates the cytokine response (Fenner et al, 1988). Such an interaction is therefore possible with smallpox and plague in people who are infected with both diseases. While there are a number of papers on the management of both smallpox and plague (Halloran, (2002), Rani et al, (2004)), there are few, if any, which discuss infection by both agents simultaneously or the likely confounding factors that will affect outcomes in their infection control after the attack. In this paper we explore the application of microsimulation modelling of a simultaneous attack on a civilian population using plague and smallpox as an example of a simultaneous coinfection through its effect on the spread of disease and number of deaths

As a basis for analysis, we have developed simulations involving a population of 1250 people based on NSW statistics for households and work. The structure of a community model of social mixing is briefly discussed, over which a multi-infection model is imposed that accounts for varying infectivity in different stages of each disease as well as confinement to home as each disease progresses. A number of simulations were run assuming 10\% immunity to both diseases, to establish a baseline for each disease in the community. Further simulations were used to model the delay of the introduction of plague compared to smallpox between 0 and 35 days respectively. The strength of the immunological interaction by smallpox on plague deaths was also investigated. Each scenario was repeated 10 times to assess the variability.

Our model showed the outcome is complex as the number of deaths is dependent on the delay in the release of plague and varies according to the number of people progressing through the active release locations that can infect them. Additionally confinement to their household during the later stages of both diseases reduces the number of susceptible people in the general community although it still allows for spread to other household members. It was found that the number of confections was about $10 \%$ of the total number infected and that the reduction in deaths from plague where the two infections were present also depended on how many smallpox cases were at the correct stage of development compared to the plague infection. We believe that the use of microsimulation has a benefit in that many of the human factors that affect delay in control and hence the size of any epidemic can be easily incorporated into the simulation without affecting the other components of the simulation. For example, vaccine and drug supply can be added as additional peeps that move to hospitals or doctors' surgeries and then interact with the human population through creation of additional states based on these locations. Further work is planned to assess the impact of these human factors on preventing epidemic spread as well as assessing current WHO guidelines for each disease. The intention is to develop protocols for application both within and outside of conflict areas. Progress on this will be reported at the conference.

Keywords: Bioterrorism, Microsimulation, Plague, Smallpox, Disease Management 


\section{INTRODUCTION}

The current conflict in Syria has raised serious international concern regarding the use of chemical and biological weapons on civilian populations in conflict zones. Reports in the media have intimated that chemical weapons have been used in the conflict $(\mathrm{BBCa}, \mathrm{BBCb}, \mathrm{BBCc})$. Because it has the potential to spread rapidly outside the conflict area, the more serious risk is the use of biological weapons. While the quality and viability of bioterrorism stockpiles are unknown, and the difficulties in weaponising them documented (Leitenberg, 2005), the concern remains that both state and non-state actors, through their state sponsors, might also have access to these weapons.

Their use against civilian populations, while being against international law, raises the question of how release in a conflict zone can be countered effectively, particularly as population movement occurs across porous borders and refugee camps have high population densities. Local and international response agencies will be stretched by a biological epidemic occurring in the context of other conflict-related issues. The principal impact will be on their ability to contact-trace cases to control the epidemic. Similarly, supplies of anti-bacterials and vaccines may not be readily available, necessitating their importation from international stockpiles and delaying their application. There have been several numerical studies of the spread and control of smallpox and plague, such as Halloran, (2002) and Rani et al, (2004), where the authors have used stochastic methods either to evaluate intervention strategies for control of outbreaks or to estimate the number of infectids ${ }^{1}$ hidden from authorities in a community once the disease outbreak had occurred. The modelled intervention strategies tended to be based on common procedures for tracing, vaccination and isolation of contacts. While many of these recommendations may work well in Western, non-conflict situations, conflict areas have problems that delay surveillance, delay access to medication and restrict the quantity and quality of trained staff. In addition, the presentation of more than one disease may confound treatments. Over the last decade a number of discrete methods have been developed that model infection spread through communities using models of social mixing. The main difference between them is how they deal with infection probability. For example, Carly et al (2003) uses risk factors for disease likelihood in different sub- populations to inform on the infection probability while Riley (2006) uses a transmission probability on contact.

In this paper we present development of our microsimulation framework, Simulacron, (Keep, 2012) to multiinfections with a preliminary investigation into the building of a model for studying the impact of multiple biological weapons releases on a population. In the work reported here we have used plague and smallpox as two indicative and plausible (Gordon, 2007) biological weapons.

\section{SIMULATION OF MULTI-INFECTION IN A COMMUNITY}

Three essential components are required to simulate multi-infection spread within a community at the level of the individual.

First, a description of the community through which the infection will spread. This includes breaking down a population into age-based and work-based compartments. The community model used in this study is based on the 2011 census data for NSW and employment statistics for NSW (ABS, 2012, NSW 2012). ${ }^{2}$ Each member of the population is assigned to a household that matches NSW housing statistics and to a location, such as a school or place of work. These locations are associated with one of six compartments that are used to assign schedules for each individual: infants, primary school and secondary school children, full time employed, part time employed and unemployed including retirees. Workplace structures conform to the overall industry sector statistics.

Second, a model of social mixing that emulates the complex patterns of locational behavior such as going to work, school or eating out at lunch or dinner. In the simulation this mixing occurs through a hierarchical structure of daily and weekly schedules. A daily schedule is chosen at random from a list of potential schedules that is dependent on the type of person and the day of the week and these are then combined into a weekly schedule. These selections for example take account of days off or sick days for full time employed or the sport activities of children on the weekend. Each daily schedule randomly selects the timing of events within time periods during the day, such as the start of work or lunch, and the place where this occurs.

\footnotetext{
${ }^{1}$ An infectid is defined as a person who has been infected with a disease and can infect others.

2 A NSW community was chosen for convenience to progress understanding of the disease processes involved. Conflict areas, however, occur all over the world and are not confined to places like Syria; for example the Cronulla riots in NSW 2005 indicate that NSW is not immune.
} 


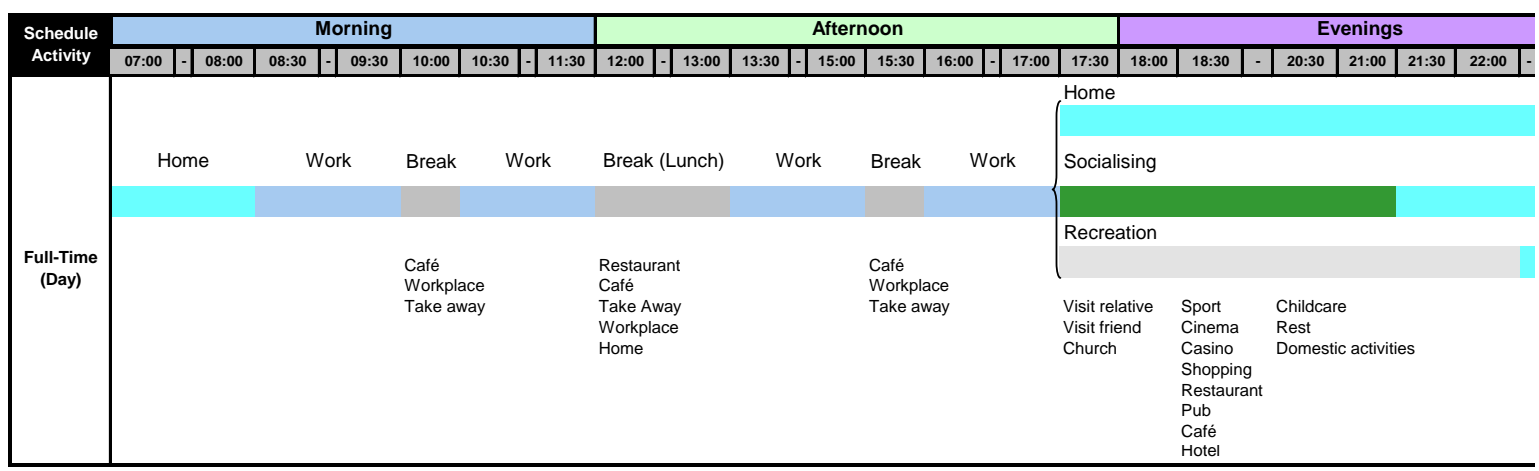

Figure 1 An example of a daily schedule.

Individuals are moved by teleportation at each event time. ${ }^{3}$ Figure 1 is an example of a daily schedule for full time work over a day. At each event time, a person has a choice of alternative activities which are preselected randomly for execution during the simulation. Complex mixing patterns occur from these very simple constructs and these patterns mimic typical social behaviours.

Third, a multi-infection module that allows spread of diseases through the community. Each infection is implemented as a discrete chain model through six infection states (Green et al., 2011). As contact time is explicitly modeled through the local social interaction, a probability of infection (infection chance) is used to determine whether the disease is spread and a probability of recovery allows transition to immunity or death states. In our model we included the ability to specify the infection probability as a relative force of infection (infection strength) by infectids as shown by equation 1 .

$A_{I C}=T_{I C} \frac{\Delta t}{t_{I}} \sum_{j=1}^{n} S_{I j}$

Where $A_{I C}$ is the infection chance at a susceptible target with infection chance, $T_{I C} . \Delta t$ is the tick time interval and $t_{I}$ is average infectious time. $S_{I j}$ is the infection strength of an infectious peep, $j$, with a compatible infection mask within the cell. The sum is over all such infectious peeps. Infection is determined by selecting a $[0,1)$ uniform random variate, and comparing it to $A_{I C}$. If the random number is less than $A_{I C}$, infection spreads to the target peep.

As the infection source is a biological weapon, it can either be dropped randomly into an area or taken by a person to a specific area of release. In this study we have randomly selected from the workplace locations to emulate the former release mode rather than the latter which would tend to be targeted at specific high density locations for maximal effect. The weapon source has its infection strength increased, compared to a natural outbreak of the disease, in order to simulate an aerosolized weapon, while subsequent spread between individuals will be similar to natural outbreaks.

As the innate immune system works in opposite directions for the two diseases, the use of additional states of infection extends the multi-infection module to allow interactions between the infections to occur. Figure 2 shows the basic infection states and their function. The potential interaction is investigated in this paper. Triggers on change of infection state are used to place individuals into states where the infections can interact within a person either increasing or decreasing the recovery rate, depending on which infection occurred first. These triggers are also used to confine individuals to one of the bedrooms in their house. While other options such as hospitalisation are possible, they have not been modelled in this work. The actual model used is more complex than that shown in Figure 2: additional states are required to take account of varying infectivity over the course of infection as discussed below.

\section{SIMULATIONS AND DISCUSSION}

The initial simulations involved a population of 1250 people in a small community. Each scenario, discussed below was simulated 10 times to understand the variability in the simulations. The two infections' states and the recovery and infection probabilities are shown in Table 1 for individuals in the simulations. Smallpox was eradicated in 1972 and persons under the age of about 40 would have no immunity. Even those over that age are unlikely to have been vaccinated except when travelling to prone areas and, in a bioweapons attack, the strain might not match the vaccination strains.

\footnotetext{
${ }^{3}$ A more detailed transport model involving path tracing, though available as a module within Simulacron, is not used in this study.
} 


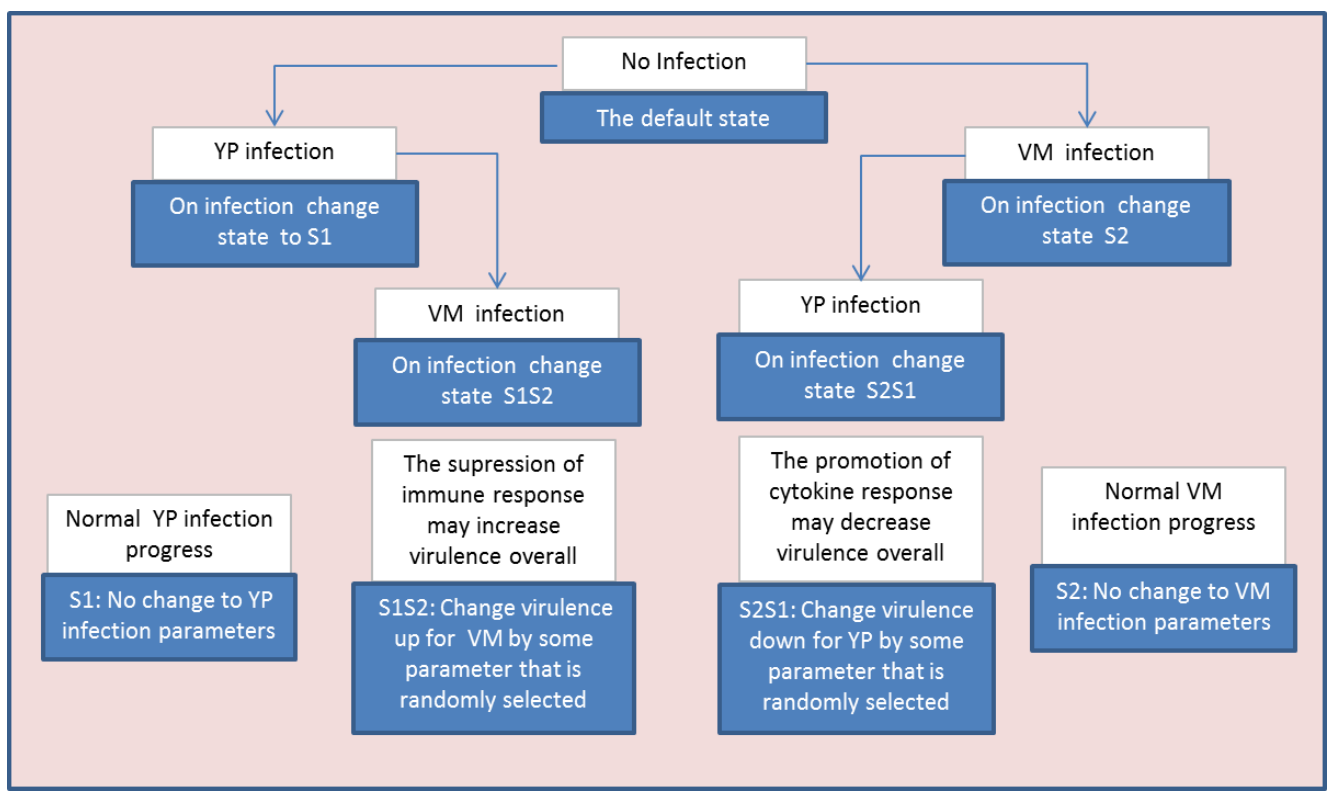

Figure 2 The State system for allowing interaction between Plague (YP) and Smallpox (VM)

The immunity to smallpox was therefore assumed to be $10 \%$ for the population as a whole. While a more detailed model of immunity could be constructed, the approach taken was considered to be reasonable given the variation in other parameters. A similar value was used for immunity to pneumonic plague on the basis of sporadic natural outbreaks around the world leaving most of the world's population vulnerable. The infection strength was varied in individuals to account for changes in infectivity during the course of the diseases. It was set at $50 \%$ during hero time ${ }^{4}$ which precedes the symptomatic time to take account of the increasing infectivity during the symptomatic phase of plague. With smallpox, the most infectious period is the prodromal period (here modelled using the asymptomatic state) and the infection strength decreases once the rash appears.

The bioweapon infections were modelled as a static person in a cell who had a latency period that matched the time from the start of the simulation to the release of the weapon's infection. This time was randomly selected from within a time window and the location was randomly selected from the workplaces in the simulation. The infectivity of the source was raised above 1.0, as shown in Table 1, to ensure a reasonable spread within the community in order that other effects of the two diseases could be studied. ${ }^{5}$ Other parameters came from analysis of outbreaks that had occurred for the two diseases.

An analysis of generational infection in an outbreak of plague in Mukden, Manchuria in 1946 (Tieh et al, 1948) was used to assess the infection probability for plague. In this outbreak 43 people were infected among 83 contacts. While the text did not explicitly state the contacts for the five that recovered, the text did allow identification of who was likely to have infected them. The infection chance for the present study is based on the average infection rate through the population in this case study.

Because the reproductive number $\left(\mathrm{R}_{0}\right)$ in the literature for smallpox varied from 0 to 38 with a mean below 3 (Bhatnagar et al., 2006), an outbreak in Abakaliki, Nigeria, in 1967 was chosen to calibrate the infection rate in a naïve population. In this outbreak, three population groups, a naïve population belonging to a faith group, a vaccinated group who lived in close proximity to the faith group and a more general population who also had a high vaccination rate were used. There was sufficient detail in the published outbreak to be able to reconstruct the populations, their immunological status and their housing communities (Thompson et al., 1968, Ottenberg, 1960). The values for the infection in Table 1 were based upon this outbreak. Simulation of the outbreak calibrated the infection chance and the other infection parameters.

\footnotetext{
${ }^{4}$ Our infection model uses 6 infection states: susceptible, latent, asymptomatic infectious, hero infectious, symptomatic infectious, immune and dead. Hero time is the infectious period where people tend to carry on as normal rather than socially distancing themselves but can be utilised more generally as an additional infectious period.

${ }^{5}$ This study is not concerned with the efficiency of weapons. Consequently, an infection chance was chosen that consistently produced spread within the community for the two diseases.
} 


\begin{tabular}{|l|l|l|}
\hline Variable & Plague & Smallpox \\
\hline Latent time & $100 \pm 40$ hours & $330 \pm 48$ hours \\
Asymptomatic (prodromal in smallpox) time & 0 hours & $50 \pm 20$ hours \\
Hero time & $<24$ hours & $48 \pm 13$ hours \\
Infectious time & $62 \pm 30$ hours & $420 \pm 60$ hours \\
Immune time (assumed) & 100000 hours & 100000 hours \\
Infection chance & $0.0022 \pm 0.0003$ & $0.01 \pm 0.002$ \\
Recovery chance & $0-0.1$ & $0.6-0.7$ \\
Infection strength of infected people & $1(0.5$ hero time) & 1 prodromal, 0.2 infectious \\
Infection strength of infection source & 1000 & 10 \\
\hline
\end{tabular}

Table 1 Infection Parameters: note that the asymptomatic time variable is used for the infectious prodromal stage of smallpox.

In the simulations, people who became infected with smallpox had a $50 \%$ chance of confinement to their home during the prodromal phase and $50 \%$ chance of confinement during the first observations of progression of the disease (Halloran, 2002). With plague there was no asymptomatic infectious stage and it was assumed that $50 \%$ of infectids would be home confined during hero time and $100 \%$ thereafter. The variation in infection chance over the course of each disease increases the number of states required to 17 compared with Figure 2.

The initial simulations were undertaken to obtain a baseline for each infection separately. Figure 4 shows the results where infection was started in five locations over a two hour period, 12 hours into the simulation. Smallpox required lower infection strengths compared to plague to obtain infection of people in the locations. While smallpox tended to spread throughout the population, plague did not spread as much and exhibited bimodal behavior. The frequency between the two modes is shown in Figure 4: about $65 \%$ of the simulations showed low propagation. This behavior seems to be relative to the number of people going through the release locations over the $2 \mathrm{hr}$ period the release was active. A larger population density might change this finding.

Figure 5 shows the plague and smallpox outbreaks when there were five release points for each infection over the same time period and the interaction was not considered. The high plague propagation has about 30 less infections on average than when it is the only disease and occurs for about 10 days longer. The smallpox numbers are very similar to the single infection numbers but the main rise in the curve occurs a few days later. Both infections are affected by home confinement reducing the susceptible population available for further infection by a second agent. There is no biological interaction between the infections in this simulation. Note that the plague still has bimodal behavior similar to the single outbreak in Figure 4, left.

A second series of simulations involved adding interaction parameters between plague and smallpox. This was achieved by having two parameters controlling the change in recovery compared to no interaction for each of the two diseases. Figure 6 shows the results for adjusting the strength of the immunological interaction when smallpox is caught first, leading to a reduction in the chance of death from plague. The simulations show that while there is a reduction in plague deaths due to the delay in the release of plague, it is not clear how the strength of the interaction between smallpox and plague is affecting the number of deaths.

Since any interaction in a double infection simulated here has to occur within a certain time period from infection with smallpox, the number of smallpox cases that would influence the outcome of plague was extracted from the simulations. In Figure 7 two sets of data are plotted against the number of plague deaths for the release of plague on the $1^{\text {st }}$ and $36^{\text {th }}$ days of simulation: firstly the number of smallpox cases that result in an interaction together with a regression estimate of the trend and secondly the effect of the reduction in the chance of death. The simulations show that the number of plague deaths depends on the delay in release of plague compared to smallpox as well as the strength of the interaction between the two diseases provided that there are enough smallpox cases where the interaction can make a difference. Increasing the number of available interactions does tend to decrease the number of deaths.

These preliminary simulations have shown that it is possible to construct a community based infection model based on individual spread of multiple diseases which allows exploration of the effects of co-infection within 
individuals. In this case the two diseases, smallpox and plague, are assumed to interact through different channels in the innate immune system leading to complex outcomes on the community when more than one biological weapon is released into a conflict zone.

Further work is planned to assess the impact of human factors on preventing epidemic spread within and outside of conflict areas. The use of microsimulation has a benefit in that many of the human factors can be easily incorporated into the simulation without affecting the other components of the simulation. For example, vaccine and drug supply can be added as additional peeps that move to hospitals or doctors' surgeries and then interact with the human population through creation of additional states based on these locations. This allows testing of policy on WHO guidelines for practitioners, for emergency preparedness and the practical steps that can be taken to reduce mortality and morbidity.

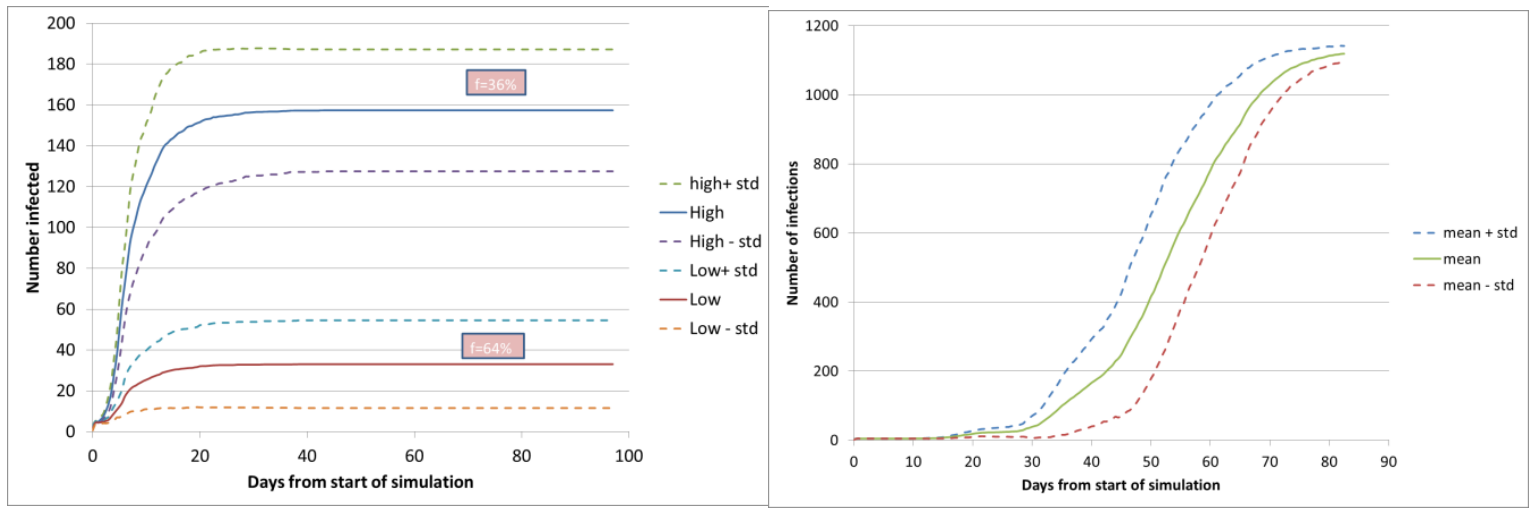

Figure 4 Individual simulation of plague (left) and smallpox (right)

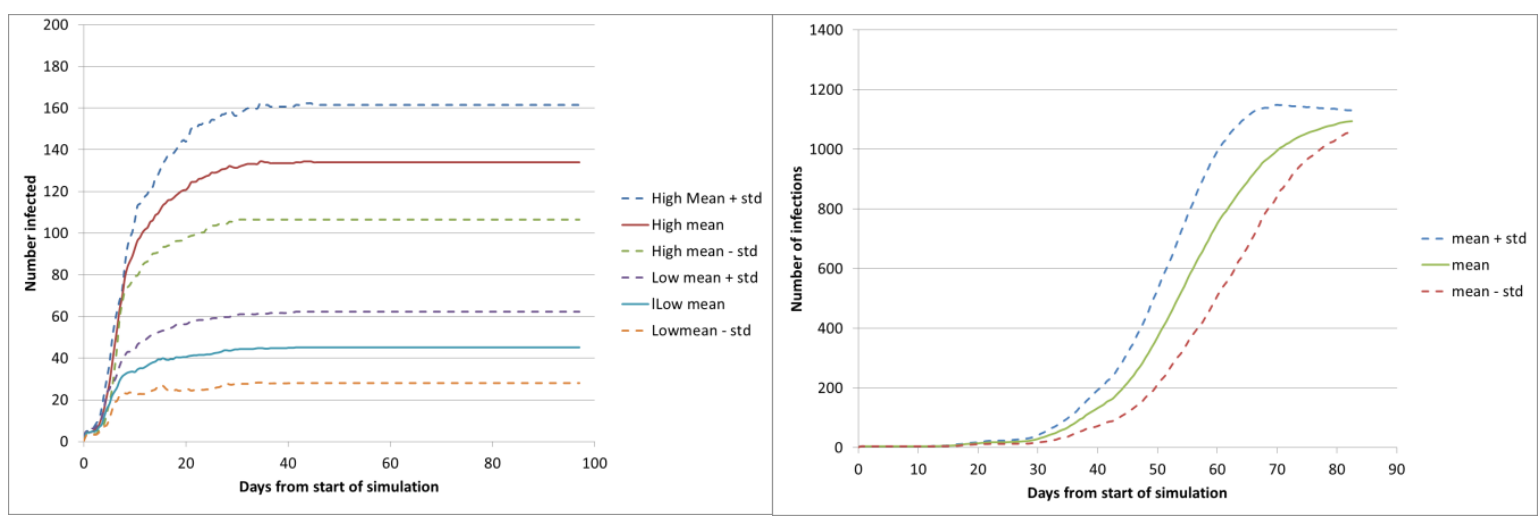

Figure 5 Simultaneous releases of plague (left) and smallpox (right)

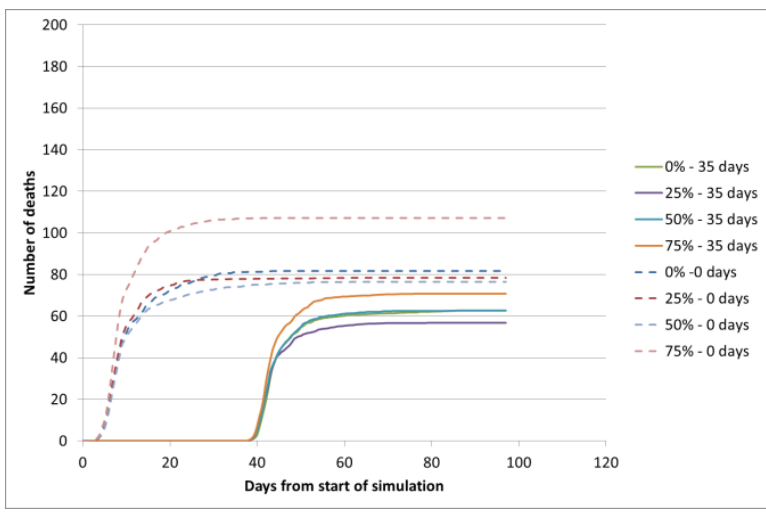

Figure 6 Deaths from Plague release 0 and 35 days after start of simulation. $\%$ is the assumed reduction in virulence of plague from co-infection.

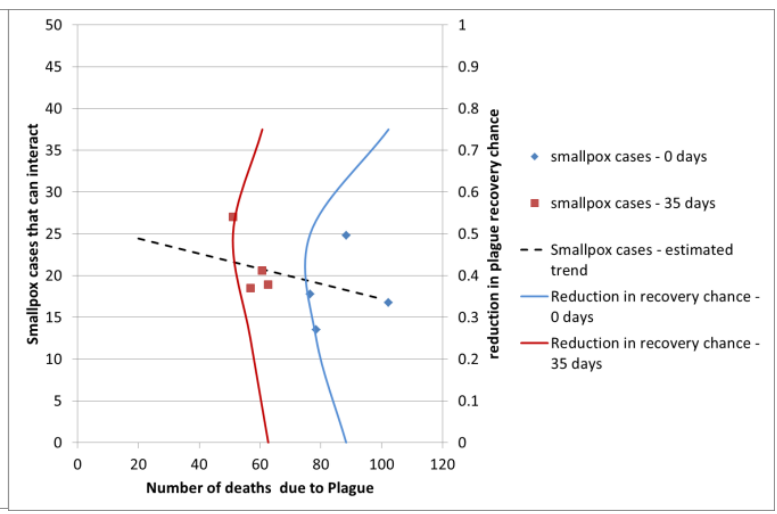

Figure 7 Number of smallpox infections that interact biologically with plague infection released at 0 and 35 days. 


\section{REFERENCES}

ABS (2012). Australian Bureau of Statistics (2011) Census data., accessed April 2013. http://www.censusdata.abs.gov.au/census_services/getproduct/census/2011/quickstat/1.

Barton, E. S., D. W. White, J. S. Cathelyn, K. A. Brett-McClellan, M. Engle, M. S. Diamond, V. L. Miller, and H. W. Virgin (2007). Herpes virus latency confers symbiotic protection from bacterial infection, Nature 447, 326-330.

BBC (2013a, b and c). Syria chemical weapons allegations. http://www.bbc.co.uk/news/world-middle-east22557347, accessed August 2013. Syria crisis: UN inspectors renew chemical attack probe. http://www.bbc.co.uk/news/world-us-canada-23845800, accessed August 2013. Syria chemical weapons: Sarin and mustard gas. http://www.bbc.co.uk/news/world-middle-east-22307705, accessed June 2013.

Bhatnagar, V., M. A. Stoto, S. C. Morton, R. Boer, and S. Bozzette (2006). Transmission patterns of smallpox: systematic review of natural outbreaks in Europe and North America since World War II. BMC Public Health 2006, 6:126, http://www.biomedcentral.com/1471-2458/6/126, accessed April 2013

Broussard, L. A. (2001). Biological agents: weapons of warfare and bioterrorism. Mol. Diagn., 6, 323-333.

Carly, K., D. Frisma, E. Casman, N. Altman, J. Chan, B. Kaminsky, D. Nave, and A. Yahja (2003). Biowar: Scalable Multi-Agent Social and Epidemiological Simulation of Bioterrorism Events. http://www.casos.cs.cmu.edu/publications/papers/carley_2003 biowarscalablemulti.pdf, accessed August 2013.

Fenner, F., D. A. Henderson, I. Arita, Z. Jezek, and I. D. Ladnyi (1988). The pathogenesis, pathology and immunology of smallpox and vaccinia. Smallpox and its eradication, World Health Organization, Geneva, 121-168. http://whqlibdoc.who.int/smallpox/9241561106_chp3.pdf, accessed April 2013.

Gani, R., and S. Leach (2004). Epidemiologic Determinants for Modelling Pneumonic Plague Outbreaks. Emerging Infectious Diseases 10(4).

Gordon, J. (2007). Syria's Bio-Warfare Threat: An Interview with Dr Jill Dekker. New English Review. http://www.newenglishreview.org/Jerry_Gordon/Syria\%27s_Bio-

Warfare Threat: an_interview_with_Dr._Jill_Dekker/, accessed April 2013.

Graham, A. L., I. M. Cattadori, J. O. Lloyd-Smith, M. J. Ferrari, and O. N. Bjornstadt (2007). Transmission Consequences of Coinfection: Cytokynes Writ Large? Trends in Parasitology 23(6), 284-29

Green, A., Y. Zhang, I. Piper, and D. Keep (2011). Application of Microsimulation to Disease Transmission and Control. In Proceedings of the 2011 MODSIM International Congress on Modelling and Simulation, pp. 919-925.

Halloran, M. E., I. M. Longini Jr., A. Nizam, and Y. Yang (2002). Containing Bioterrorist Smallpox. Science 298, 1428-1432.

Keep, D. R. (2012). Generalised Microsimulation. Ph. D. thesis, University of Wollongong.

Lane, H. C., J. L. Montagne, and A. S. Fauci (2001). Bioterrorism: a clear and present danger. Nature Medicine 7, 1271-1273.

Leitenberg, M. (2006). Assessing The Biological Weapons And Bioterrorism Threat. Department of the Army, Department of Defense, U.S. Government. ISBN 1-58487-221-7.

Li, B., and R. Yang (2008). Interaction between Yersinia Pestis and the Host Immune System. Infection And Immunity 76(5), 1804-1811.

NSW (2012). Workforce Data. http://www.abs.gov.au/ausstats/abs@.nsf/Lookup/2901.0Chapter38702011, accessed April 2013.

Ottenberg, S. (1960). Abakaliki photographs. Eliot Elisofon Photographic Archives, National Museum of African Art, Smithsonian Institution, http://siris-archives.si.edu/ipac20/ipac.jsp?uri=full=3100001 !224045!0\&term=, accessed April 2013

Thompson, D., and W. Foege (1968). Faith Tabernacle Smallpox Epidemic Abakaliki, Nigeria. World Health Organisation. http://apps.who.int/iris/bitstream/10665/67462/1/WHO_SE_68.3.pdf, accessed April 2013.

Tieh, T. H., E. Landauer, F. Miyagawa, G. Kobayashi, and G. Okayasu (1948). Primary Pneumonic Plague In Mukden, 1946, And Report Of 39 Cases With 3 Recoveries. Journal of Infectious Diseases 82(1), 52-58. 